\title{
Ischemia modified albumin levels in intrauterine growth restriction: levels are increased in fetal cord blood but not in maternal blood
}

\author{
Ceren Gölbaşı1 ${ }^{1} \mathbb{D}$, Hakan Gölbaşı ${ }^{\mathbb{D}}$, Ceysu Kocahakimoğlu Gültekin², Varol Gülseren² ${ }^{\mathbb{D}}$, \\ Merve Zeytinli Akşit ${ }^{3}$ D , Burak Bayraktar² ${ }^{\mathbb{D}}$, Ayfer Çolak ${ }^{3}$ (D) , Cüneyt Eftal Taner $^{2}$ (D) \\ ${ }^{1}$ Department of Obstetrics and Gynecology, Izmir Tınaztepe University Faculty of Medicine, Izmir, Turkey \\ ${ }^{2}$ Department of Obstetrics and Gynecology, University of Health Sciences Tepecik Training and Research Hospital, Izmir, Turkey \\ ${ }^{3}$ Department of Biochemistry, University of Health Sciences Tepecik Training and Research Hospital, Izmir, Turkey
}

\begin{abstract}
Objectives: In this study, our aim was to determine the differences between intrauterine growth restriction (IUGR) and normal birth weight fetuses in terms of ischemia modified albumin (IMA) levels. For this purpose, we measured ischemia modified albumin levels in the cord blood of fetuses and venous blood of mothers.

Material and methods: This study is a prospective study conducted at University of Health Sciences Tepecik Training and Research Hospital between January 2018 and December 2019. According to the inclusion/exclusion criteria, 227 patients were included in the study. Participants were divided into two groups according to the presence (patient group) or absence (control group) of intrauterine growth restriction (IUGR). In addition to routine parameters recorded during pregnancy, the IMA levels and Doppler USG findings of all participants were recorded.

Results: The mean cord blood serum IMA levels of the patient group were significantly elevated compared to controls $(p=0.038)$. Whereas mean maternal blood serum IMA levels were similar among groups $(p=0.453)$. The cord blood and mother blood serum IMA levels were not significantly different with regard to the presence or absence of perinatal asphyxia.

Conclusions: In the literature, studies evaluating IMA levels in the cord and maternal blood of IUGR fetuses are limited. In IUGR patients, IMA level is expected to increase and in our study, IMA levels were significantly increased but the presence of oxidative stress has not been found to affect IMA levels.
\end{abstract}

Key words: ischemia modified albumin (IMA); oxidative stress; intrauterine growth restriction (IUGR); cord blood; asphyxia

Ginekologia Polska 2022; 93, 12: 993-998

\section{INTRODUCTION}

Ischemia Modified Albumin (IMA) is a non-specific ischemia marker that is associated with insufficient oxygenation, elevated anaerobic metabolism, and free oxygen radicals [1]. The clinical utility of IMA has been demonstrated in the identification of acute coronary syndrome [2, 3]. It was a marker that received significant interest by researchers and clinicians as it was believed to show ischemia in its early stages; that is before necrosis occurred. In addition, it has been explored in diseases such as diabetes mellitus, trauma, heart failure, and pulmonary embolism [4, 5]. The interesting results put forth in these studies and the fact that IMA measurement is very cheap have attracted the at- tention of various other specialties, including perinatologists and gynecologists, for its use as a marker of ischemia [6].

Although there are various opinions about the approach to the delivery of low birth weight infants and when infants with intrauterine growth restriction (IUGR) should be delivered $[7,8]$, each case is multifactorial and should be an individual decision based on many factors. The current problem with timing is to prevent intrauterine fetal loss while also allowing sufficient time for fetal lung development. The hypoxic environment in which fetuses with intrauterine growth retardation are situated can lead to growth retardation and negative effects on brain development. Early detection of hypoxia is critical when considering the possibility 
of preventing such problems. Several markers reflecting the oxidative stress of the fetus, such as prolidase activity, total free sulfhydryl and various parameters in the oxidative stress index have been studied for this purpose [9]. In addition to well-established markers, some researchers have investigated whether IMA can be used to determine intrauterine hypoxia $[6,10,11]$. Moreover, studies have been conducted previously on the measurement of IMA in conditions such as preeclampsia, preterm delivery, and recurrent miscarriages [12-14]. Most field studies have been done by measuring IMA levels in cord blood; however, maternal blood levels of this marker may also be important for fetal development. On the other hand, it is apparent that the number of patients included in these studies and the number of studies themselves are insufficient to reach any clear conclusion as to how IMA levels change in such conditions [6].

In this study, our aim was to ascertain whether IMA levels could be regarded as a marker that could identify the optimal birth time in fetuses with IUGR. For this purpose, we measured and compared IMA levels in the cord blood and maternal circulation of all patients who accepted to participate in the study, and we evaluated results according to birth weight.

\section{MATERIAL AND METHODS}

\section{Study Group}

This is a prospective study conducted at University of Health Sciences Tepecik Training and Research Hospital from January 2018 to December 2019. The criteria for inclusion in the study were to give birth between the age of 18-40 years, accepting to participate in the study and to attend all pregnancy control evaluations at our hospital. The exclusion criteria were:

- Multiple pregnancies (7 individuals excluded)

- Fetal malformation or chromosomal anomaly (3 individuals excluded)

- Delivery with complications (dystocia, cord prolapse, placental abruption) ( 6 individuals excluded)

- Neonatal complications (11 individuals excluded)

- Gestational diabetes mellitus (16 individuals excluded)

- Preeclampsia (21 individuals excluded)

- Recurrent pregnancy loss (3 individuals excluded)

A total of 294 women who met our criteria gave birth during the study. According to the above-mentioned reasons, 67 woman were excluded. As a result, a final group of 227 individuals were included in the study and were divided into two groups according to fetal birth weight. Those with low birth weight comprised the patient group $(n=76)$, while those with normal birth weight formed the control group ( $n=151$ ). In addition, patients were divided into groups according to the presence/absence of asphyxia and the effect of oxidative stress was evaluated.
Fetuses with an expected fetal weight below the $10^{\text {th }}$ percentile threshold according to gestational age were accepted to have IUGR when gestational age could be determined accurately and there were no signs of constitutionally small stature (regarding maternal and paternal characteristics). Perinatal asphyxia was defined as the occurrence of asphyxia in the newborn after birth and diagnosis was made according to the criteria of American Academy of Pediatrics (AAP) and American College of Obstetrics and Gynecology (ACOG) $[13,15]$ : (i) metabolic acidosis in fetal umbilical cord arterial blood obtained at delivery $(\mathrm{pH}$ less than 7 and base deficit of $12 \mathrm{mmol} / \mathrm{L}$ or more, (ii) persistence of an Apgar score of 0-3 for longer than 5 minute, (iii) neonatal neurologic sequelae (e.g., seizures, coma, hypotonia), and (iv) multiple organ involvement (e.g., kidney, lungs, liver, heart, intestines).

\section{Ethical}

Permission for the study was obtained from the Clinical Research Ethics Committee of University of Health Sciences Tepecik Training and Research Hospital. During the conduct of the study, the Helsinki Declaration and Good Clinical Practices Guide were followed. Written and oral consent was obtained from all mothers who participated in the study.

\section{Measurements}

All routine data obtained to assess gestational health (including maternal age, gestational age, gravida, parity, birth weight, fetus gender, type of delivery, history of fetal death) were recorded for the study. Blood samples from the umbilical vein (after clamping of the cord) were drawn into serum separator tubes immediately after delivery.

The cerebroplacental ratio (CPR) was determined by the measurement of umbilical and middle cerebral artery pulsatility indexes (PI) via Doppler. The middle cerebral artery (MCA) PI values were calculated via measurements performed at the portion of the artery that passes close to the sphenoid wing, while the umbilical artery (UA) PI values were calculated from measurements performed at the free loop. During measurements, the insonation angle was kept lower than $30^{\circ}$ and values were recorded when fetal movements were absent. The cerebroplacental ratio (CPR) was defined as the ratio between middle cerebral artery (MCA) PI and umbilical artery (UA) PI. All results obtained with this procedure were converted into multiples of the median (MoM), with corrections performed according to gestational age reference ranges [16].

Cesarean sections were carried out with the following protocol: The patients did not receive premedication therapy. In the operating room, patients were placed supine before. Then, oxygen (3L/min) and intravenous colloidal solution ( $20 \mathrm{~mL} / \mathrm{kg} /$ hour) were given to the patients. After 
three minutes in this state, baseline measurements (Oxy-, Deoxy-, Total-Hb Concentrations, Tissue Oxygenation Index, Mean Blood Pressure, and Heart Rate) were recorded. Later, the patients were placed in the lateral position and lumbar puncture was performed at the L3/L4 interval. Hyperbaric $0.5 \%$ bupivacaine was used in all patients by adjusting the individual dosage by the anesthesiologist. After the injection, the patients were placed in the supine position. If anesthesia-related hypotension was observed (MBP $<80 \%$ of baseline value or systolic blood pressure $<90 \mathrm{mmHg}$ ), ephedrine was given to the patient by the anesthesiologist.

Measurement of IMA was performed spectrophotometrically according to the method described by Bar-Or et al. [17]. According to this method, $200 \mu \mathrm{L}$ patient blood serum was mixed with $50 \mu \mathrm{L}$ of $0.1 \%$ cobalt chloride (Sigma, St. Louis, MO, USA) and incubated for 10 minutes. After the incubation step, $50 \mu \mathrm{L}$ of dithiothreitol (DTT) solution in water $(1.5 \mathrm{mg} / \mathrm{mL}$ ) (Sigma, St. Louis, MO, USA) is added. After 2 minutes, $1 \mathrm{~mL}$ of $0.9 \% \mathrm{NaCl}$ is added. The absorbance of the sample is measured with a spectrophotometer set at $470 \mathrm{~nm}$. A sample without DTT addition is used as the blank for each measurement. All results were reported in absorbance units (AbsU).

Fetal and maternal IMA measurements were performed at $>37$ weeks of gestation.

\section{Statistical analysis}

Power analysis was performed with G-Power 3.1.9.7. Accordingly, the total minimum number of people to attend was calculated as 46 for each group. SPSS version 22.0 (IBM Corporation, Armonk, New York, US) was used for statistics and calculations. Differences between cases and controls were tested for significance using the chi-square test for categorical variables. The normally distributed variables were evaluated using Shapiro-Wilk tests. Independent T-test was used if the data showed normal distribution for continuous variables (data were presented as mean \pm standard deviation) and Mann-Whitney $U$ test was used if the data did not show normal distribution (data were presented as median $\pm \min , \max ) . p<0.05$ was accepted as statistically significant.

\section{RESULTS}

Seventy-six (33.5\%) of the 227 neonates had IUGR. Gestational ages and birth weights were lower in the IUGR group compared to the newborns in the control group. However, no differences were found in maternal age between these groups. In terms of IMA, the mean cord blood serum IMA levels of the patient group were significantly elevated compared to those from uncomplicated pregnancies $(p=0.038)$. However, mean maternal blood serum IMA levels were simi$\operatorname{lar}(\mathrm{p}=0.453)$. (Tab. 1).

Table 1. Clinical and demographic features of the study group

\begin{tabular}{|c|c|c|c|}
\hline & IUGR Group ( $n=76$ ) & Control Group $(n=151)$ & p \\
\hline $\begin{array}{l}\text { Fetus gender; n (\%) } \\
\text { Female } \\
\text { Male }\end{array}$ & $\begin{array}{l}49(64.5) \\
27(35.5)\end{array}$ & $\begin{array}{l}74(49.0) \\
77(51.0)\end{array}$ & 0.019 \\
\hline Maternal age; years (mean $\pm S D$ ) & $26.0 \pm 5.9$ & $27.2 \pm 6.4$ & 0.165 \\
\hline Gravida (median, min-max) & $2(1-7)$ & $2(1-10)$ & 0.044 \\
\hline Parity (mean \pm SD) & $1.7(0-6)$ & $2.2(0-10)$ & 0.002 \\
\hline Birth weight; $g$ (mean \pm SD) & $2322 \pm 546$ & $3288 \pm 376$ & $<0.001$ \\
\hline Gestational age; weeks (mean \pm SD) & $37.4 \pm 2.1$ & $38.9 \pm 1.1$ & $<0.001$ \\
\hline History of fetus mortality; $\mathrm{n}(\%)$ & $0(0.0)$ & $3(2.0)$ & 0.292 \\
\hline $\begin{array}{l}\text { History of abortion; } \mathrm{n}(\%) \\
0 \\
1 \\
2 \\
\geq 3\end{array}$ & $\begin{array}{l}60(78.9) \\
10(13.2) \\
2(2.6) \\
4(5.3)\end{array}$ & $\begin{array}{l}128(84.8) \\
14(9.3) \\
6(4.0) \\
3(2.0)\end{array}$ & 0.400 \\
\hline Amniotic fluid index (AFI); mm (mean \pm SD) & $79.5 \pm 29.7$ & $99.9 \pm 16.9$ & $<0.001$ \\
\hline Oligohydramniosis; n (\%) & $15(19.7)$ & $2(1.3)$ & $<0.001$ \\
\hline Asphyxia; n (\%) & $27(35.5)$ & $3(2.0)$ & $<0.001$ \\
\hline IMA level in cord blood (AbsU) (mean \pm SD) & $0.6 \pm 0.4$ & $0.5 \pm 0.3$ & 0.038 \\
\hline IMA level in maternal blood (AbsU) (mean \pm SD) & $0.4 \pm 0.1$ & $0.4 \pm 0.2$ & 0.453 \\
\hline Apgar (1 min) (mean \pm SD) & $6.9 \pm 0.7$ & $7.3 \pm 0.6$ & $<0.001$ \\
\hline
\end{tabular}

IMA - Ischemia modified albumin; IUGR — intrauterine growth restriction; SD — standard deviation 


\begin{tabular}{|c|c|c|c|}
\hline & Asphyxia Present $(n=30)$ & Asphyxia Absent $(n=197)$ & $\mathbf{p}$ \\
\hline $\begin{array}{l}\text { Fetal gender; } \mathrm{n}(\%) \\
\text { Female } \\
\text { Male }\end{array}$ & $\begin{array}{l}13(43.3) \\
17(56.7)\end{array}$ & $\begin{array}{l}110(55.8) \\
87(44.2)\end{array}$ & 0.139 \\
\hline Maternal age; years (mean \pm SD) & $26.0 \pm 6.0$ & $27.0 \pm 6.3$ & 0.417 \\
\hline Gravida (mean \pm SD) & $1.8(1-9)$ & $2.5(1-10)$ & 0.020 \\
\hline Parity (mean \pm SD) & $1.4(0-7)$ & $2.1(0-10)$ & 0.008 \\
\hline Birth weight; g (mean \pm SD) & $2017 \pm 683$ & $3107 \pm 486$ & $<0.001$ \\
\hline Gestational age; weeks (mean \pm SD) & $37.9 \pm 2.1$ & $38.4 \pm 1.6$ & 0.087 \\
\hline History of mortal fetus; n (\%) & $0(0.0)$ & $3(1.5)$ & 0.652 \\
\hline $\begin{array}{l}\text { History of abortion; } \mathrm{n}(\%) \\
0 \\
1 \\
2 \\
\geq 3\end{array}$ & $\begin{array}{l}25(83.3) \\
4(13.3) \\
0(0) \\
1(3.3)\end{array}$ & $\begin{array}{l}163(82.7) \\
20(10.2) \\
8(4.1) \\
6(3.0)\end{array}$ & 0.688 \\
\hline Amniotic fluid index (AFI); mm (mean $\pm S D)$ & $76.0 \pm 31.4$ & $95.7 \pm 21.5$ & $<0.001$ \\
\hline Oligohydramnios; n (\%) & $7(23.3)$ & $10(5.1)$ & 0.003 \\
\hline IMA level in cord blood (AbsU) (mean \pm SD) & $0.6 \pm 0.4$ & $0.6 \pm 0.4$ & 0.925 \\
\hline IMA level in maternal blood (AbsU) (mean \pm SD) & $0.4 \pm 0.1$ & $0.4 \pm 0.2$ & 0.181 \\
\hline Apgar (1 min) (mean \pm SD) & $6.4 \pm 1.3$ & $7.3 \pm 0.4$ & $<0.001$ \\
\hline
\end{tabular}

IMA - Ischemia modified albumin; SD — standard deviation

Table 3. Distribution of antenatal Doppler findings in 76 newborns with intrauterine growth restriction and comparison with asphyxia and non-asphyxia pregnancies

\begin{tabular}{|l|l|l|l|}
\hline & Asphyxia Present $\mathbf{( n = 2 7 )}$ & Asphyxia Absent $(\mathbf{n}=\mathbf{4 9})$ & $\mathbf{p}$ \\
\hline MCA-PI (mean \pm SD) & $1.1 \pm 0.4$ & $1.2 \pm 0.3$ & 0.426 \\
\hline MCA-S/D (mean \pm SD) & $3.4 \pm 1.2$ & $3.7 \pm 0.9$ & 0.272 \\
\hline UA-PI (mean \pm SD) & $0.9 \pm 0.2$ & $0.9 \pm 0.3$ & 0.445 \\
\hline UA-S/D (mean \pm SD) & $3.02 \pm 1.1$ & $2.90 \pm 0.8$ & 0.592 \\
\hline UA-S/D end-diastolic flow loss; $n$ (\%) & $3(11.1)$ & $3(6.1)$ & 0.851 \\
\hline $\begin{array}{l}\text { C/P Ratio (mean } \pm \text { SD) } \\
\text { C/P Ratio } n(\%)\end{array}$ & $1.2 \pm 0.5$ & $1.4 \pm 0.5$ & 0.333 \\
\hline $\begin{array}{l}\text { Normal } \\
\text { Abnormal }\end{array}$ & $17(62.9)$ & $29(59.1)$ & 0.747 \\
\hline
\end{tabular}

UA — umbilical artery; MCA — middle cerebral artery; PI — pulsatility index; C/P ratio — cerebro-placental ratio; SD — standard deviation

Thirty (13.2\%) newborns were diagnosed with perinatal asphyxia. In these cases, mean birth weight was significantly lower than the controls. Oligohydramnios before delivery was more common in those with perinatal asphyxia. The cord blood and maternal blood serum IMA levels were not significantly different in newborns with perinatal asphyxia compared to healthy controls (Tab. 2).

We also investigated Doppler findings of 76 fetuses with fetal growth retardation were compared according to the presence/absence of asphyxia. The mean C/P ratio was statistically similar between the groups. The PI values of the middle cerebral artery and the umbilical artery were also similar when those with and without asphyxia were compared. (Tab. 3)

\section{DISCUSSION}

In our study, IMA levels in the maternal blood of IUGR fetuses were found to be similar to IMA levels in the blood samples of mothers with normal fetuses. IMA concentrations were found to be higher in the cord blood samples of the IUGR group. On the other hand, it was observed that IMA levels were similar when the groups were separated according to the presence of asphyxia.

In recent years, there has been an opinion that IMA levels in cord blood are a reliable indicator of intrauterine ischemia 
and hypoxia [18]. Guvendag Guven et al. [19] compared IMA levels with the cord blood of 20 IUGR fetuses and 20 controls. The IMA levels of those with IUGR were reported to be lower in addition to significant alterations in other important parameters. They also found a strong positive correlation between umbilical artery PI and IMA levels. However, the small sample size raises concerns about the reliability of the findings of this study. In another case-control study, Gugliucci et al. [18] showed that IMA levels would increase by 3-fold in a fetus that experienced oxidative stress and hypoxia compared to healthy controls. However, the number of patients in this study is also small. In another study, which included a relatively higher number of patients (57 IUGR and 110 fetuses with normal birth weight), lakovou et al. [10] did not find a significant difference between the two groups in terms of IMA levels. However, in this study, IUGR patients were not separated according to the presence or absence of asphyxia. In our study involving 257 fetuses, although IMA levels were higher in the cord blood IUGR group, IMA levels in the cord blood of fetuses who experienced oxidative stress were similar. This suggests that IMA may be elevated in cord blood due to another reason. In this respect, the pathophysiology underlying the elevation of IMA in this condition requires elucidation.

There are a limited number of studies looking at IMA levels in samples taken from the maternal blood in cases where developmental problems are observed. Although IMA has been reported to be higher than normal pregnancies in many clinical situations, there are no studies analyzing IMA in maternal blood of IUGR fetuses. In our study, maternal IMA levels were similar in mothers with IUGR fetuses compared to mothers that had fetuses with normal development. In addition, IMA concentrations of the mothers bearing fetuses exposed to oxidative stress according to Doppler USG were found to be similar to those bearing fetuses without exposure to oxidative stress. In a study by Rossi and colleagues [11] which was comprised of 65 normal and 16 small-for-gestational-age (SGA) fetuses at different periods of pregnancy, the IMA/albumin ratio was investigated. Interestingly, while the IMA/albumin ratio was found to be higher in mothers who had SGA fetuses in the first and second trimester, the IMA/albumin ratio was similar two weeks after termination of pregnancy. Thus, the authors suggested that IMA may be useful in predicting oxidative stress in the early stages of pregnancy but may lose its sensitivity thereafter. In several studies, it was emphasized that IMA increased physiologically during pregnancy $[20,21]$.

Although our study reached some important findings, we should note that there are several limitations. First, some parameters that were likely to affect IMA levels were not similar in both groups. IMA levels might be affected by various factors, which may lead to unreliable results and conclusions. For instance, the gestational age was higher in the control group, which may have marginally affected results. In addition, being a tertiary center may have affected the high-risk pregnancy rates and the prevalence of neonatal asphyxia. We also did not look at other oxidative stress parameters which may have been useful in determining the levels of oxidative stress. Finally, we could not analyze the long-term results of our patients due to insufficient records and patients not attending long-term follow-up studies.

However, our study also has some strengths to be mentioned. Firstly, we analyzed patients regarding the presence/absence of oxidative stress. Secondly, we included a relatively high number of patients compared to other studies in a prospective fashion. Thirdly, we measured IMA in maternal blood which is absent in the majority of studies in this field. In addition, we excluded patients with clinical conditions that are known to affect IMA levels and our study group was homogeneous in terms of other confounding factors.

\section{CONCLUSIONS}

In the literature, studies evaluating IMA levels in the cord blood and maternal blood with IUGR fetuses are quite limited. Although the common opinion is that IMA levels will increase in patients with IUGR, we found that IMA levels are elevated in IUGR's and do not change compared to normal pregnancies in the presence of oxidative stress. Further studies in larger groups are needed to establish clear evidence on the subject and to determine the pathophysiologic mechanism by which IMA levels are altered.

\section{Funding}

No funding for this study.

\section{Conflict of interest}

All authors declare no conflict of interest.

\section{REFERENCES}

1. Sinha MK, Roy D, Gaze DC, et al. Role of "Ischemia modified albumin", a new biochemical marker of myocardial ischaemia, in the early diagnosis of acute coronary syndromes. Emerg Med J. 2004; 21(1): 29-34, doi: 10.1136/emj.2003.006007, indexed in Pubmed: 14734370.

2. Apple FS, Wu AHB, Mair J, et al. Committee on Standardization of Markers of Cardiac Damage of the IFCC. Future biomarkers for detection of ischemia and risk stratification in acute coronary syndrome. Clin Chem. 2005; 51(5): 810-824, doi: 10.1373/clinchem.2004.046292, indexed in Pubmed: 15774573.

3. Lippi G, Montagnana M, Guidi GC. Albumin cobalt binding and ischemia modified albumin generation: an endogenous response to ischemia? Int J Cardiol. 2006; 108(3): 410-411, doi: 10.1016/j.ijcard.2005.03.040, indexed in Pubmed: 16520132.

4. Turedi S, Gunduz A, Mentese A, et al. Value of ischemia-modified albumin in the diagnosis of pulmonary embolism. Am J Emerg Med. 2007; 25(7): 770-773, doi: 10.1016/j.ajem.2006.12.013, indexed in Pubmed: 17870479.

5. Piwowar A, Knapik-Kordecka M, Warwas M. Ischemia-modified albumin level in type 2 diabetes mellitus - Preliminary report. Dis Markers. 2008; 
24(6): 311-317, doi: 10.1155/2008/784313, indexed in Pubmed: 18688079.

6. Yarcı Gursoy A, Caglar GS, Demirtas S. Ischemia modified albumin in perinatology. Eur J Obstet Gynecol Reprod Biol. 2017; 210: 182-188, doi: 10.1016/j.ejogrb.2016.12.022, indexed in Pubmed: 28056434.

7. Lees CC, Stampalija T, Baschat A, et al. ISUOG Practice Guidelines: diagnosis and management of small-for-gestational-age fetus and fetal growth restriction. Ultrasound Obstet Gynecol. 2020; 56(2): 298-312, doi: 10.1002/uog.22134, indexed in Pubmed: 32738107.

8. Fetal Growth Restriction. Obstetrics \& Gynecology. 2021; 137(2): e16-e28, doi: 10.1097/aog.0000000000004251.

9. Toy H, Camuzcuoglu H, Arioz DT, et al. Serum prolidase activity and oxidative stress markers in pregnancies with intrauterine growth restricted infants. J Obstet Gynaecol Res. 2009; 35(6): 1047-1053, doi: 10.1111/j.1447-0756.2009.01063.x, indexed in Pubmed: 20144170.

10. lacovidou N, Briana DD, Boutsikou M, et al. Cord blood ischemia-modified albumin levels in normal and intrauterine growth restricted pregnancies. Mediators Inflamm. 2008; 2008: 523081, doi: 10.1155/2008/523081, indexed in Pubmed: 18483569.

11. Rossi A, Bortolotti N, Vescovo $S$, et al. Ischemia-modified albumin in pregnancy. Eur J Obstet Gynecol Reprod Biol. 2013; 170(2): 348-351, doi: 10.1016/j.ejogrb.2013.06.037, indexed in Pubmed: 23891063.

12. $\mathrm{Ma} \mathrm{Sg}, \mathrm{Yu} \mathrm{Wn}$, Jin $\mathrm{Y}$, et al. Evaluation of serum ischemia-modified albumin levels in pregnant women with and without gestational diabetes mellitus. Gynecol Endocrinol. 2012; 28(11): 837-840, doi: 10.3109/09513590.2012.683069, indexed in Pubmed: 22571721.

13. Gafsou B, Lefèvre $G$, Hennache $B$, et al. Maternal serum ischemia-modified albumin: a biomarker to distinguish between normal pregnancy and preeclampsia? Hypertens Pregnancy. 2010; 29(1): 101-111, doi: 10.3109/10641950902968601, indexed in Pubmed: 20132025.
14. Özdemir S, Kıyıcı A, Balci O, et al. Assessment of ischemia-modified albumin level in patients with recurrent pregnancy loss during the first trimester. Eur J Obstet Gynecol Reprod Biol. 2011; 155(2): 209-212, doi: 10.1016/j.ejogrb.2010.12.004, indexed in Pubmed: 21185113.

15. Use and Abuse of the Apgar Score. Pediatrics. 1996; 98(1): 141-142, doi: 10.1542/peds.98.1.141.

16. Bhide A, Acharya G, Bilardo CM, et al. ISUOG practice guidelines: use of Doppler ultrasonography in obstetrics. Ultrasound Obstet Gynecol. 2013, 41(2): 233-239, doi: 10.1002/uog.12371, indexed in Pubmed: 23371348.

17. Bar-Or D, Lau E, Winkler JV. A novel assay for cobalt-albumin binding and its potential as a marker for myocardial ischemia-a preliminary report. J Emerg Med. 2000; 19(4): 311-315, doi: 10.1016/s07364679(00)00255-9, indexed in Pubmed: 11074321.

18. Gugliucci A, Hermo R, Monroy C, et al. Ischemia-modified albumin levels in cord blood: a case-control study in uncomplicated and complicated deliveries. Clin Chim Acta. 2005; 362(1-2): 155-160, doi: 10.1016/j. cccn.2005.06.014, indexed in Pubmed: 16018994.

19. Guvendag Guven ES, Karcaaltincaba D, Kandemir O, et al. Cord blood oxidative stress markers correlate with umbilical artery pulsatility in fetal growth restriction. J Matern Fetal Neonatal Med. 2013; 26(6): 576-580, doi: 10.3109/14767058.2012.745497, indexed in Pubmed: 23130645.

20. Cengiz $H$, Dagdeviren $H$, Kanawati $A$, et al. Ischemia-modified albumin as an oxidative stress biomarker in early pregnancy loss. J Matern Fetal Neonatal Med. 2016; 29(11): 1754-1757, doi: 10.3109/14767058.2015.1061494, indexed in Pubmed: 26135770.

21. Prefumo F, Gaze DC, Papageorghiou AT, et al. First trimester maternal serum ischaemia-modified albumin: a marker of hypoxia-ischaemia-driven early trophoblast development. Hum Reprod. 2007; 22(7): 2029-2032, doi: 10.1093/humrep/dem095, indexed in Pubmed: 17437959. 